\title{
Numerical Investigation of Heat and Mass Transfer Processes while the Desorption of Hydrogen Gas Stored in $\mathrm{MmNi}_{4.6} \mathrm{Fe}_{0.4}-\mathrm{H}_{6}$
}

\author{
Ali Boukhari \\ Department of Mechanical Engineering, Faculty of Technology, University of El-Oued 39000. Algeria. \\ E-Mail: fibonali2379@gmail.com
}

\begin{abstract}
This work presents a numerical investigation of two-dimensional coupled heat and mass transfer processes in a unit disc of an annulus-disc reactor filled with the intermetallic (Mischmetal) compound $\mathrm{MmNi}_{4.6} \mathrm{Fe}_{0.4}$, during the hydrogen gas desorption using the finite volume method. Temperature and amount of desorbed hydrogen and their time-averaged quantities inside the metal hydride bed are presented for different heat transfer fluid temperatures, and different metal thermal conductivities. Impacts of both effects on the metal hydride reactor performance in terms of discharging time are examined by means of a set of numerical simulations. Thus, the dehydriding time minimization relates to the adjustment of the amount of heat addition to the packed bed reactor. A good agreement was found between the present computational results and the experimental data reported in the literature.
\end{abstract}

KEYWORDS - Numerical simulation, Coupled heat and mass transfer, Finite volume method, Metal hydride, Desorption.

\section{NOMENCLATURE}

$\begin{array}{ll}A, B & \text { van't Hoff constants } \\ b & \text { Forchheimer's coefficient, } \mathrm{m}^{-1} \\ C_{d} & \text { desorption rate coefficient, } \mathrm{s}^{-1} \\ C_{p} & \text { specific heat, } \mathrm{J} / \mathrm{kg} \mathrm{K} \\ d_{p} & \text { particle diameter, } \mathrm{m} \\ E_{d} & \text { desorption activation energy, } \mathrm{J} / \mathrm{mol} \\ h & \text { heat convection coefficient, } \mathrm{W} / \mathrm{m}^{2} \mathrm{~K} \\ K & \text { permeability, }{ }^{2} \\ \dot{m} & \mathrm{H}_{2} \text { mass reaction rate, } \mathrm{kg} / \mathrm{m}^{3} \\ P & \text { pressure, Pa } \\ \mathfrak{R} & \text { universal gas constant, } \mathrm{J} / \mathrm{mol} \mathrm{K} \\ R & \text { radius of the annular disc unit, } \mathrm{m} \\ r & \text { radius of cooling tube, } \mathrm{m} \\ S_{D x}, S_{D y} & \text { Forchheimer terms in the } x \text { and } y \text {-directions } \\ T & \text { temperature, } \mathrm{K} \\ t & \text { time, } \mathrm{s} \\ \vec{V} & \text { velocity in the metal bed, } \mathrm{m} / \mathrm{s} \\ w t . \% & \text { hydrogen storage capacity } \\ x, y & x \text { and } y \text {-coordinates }\end{array}$

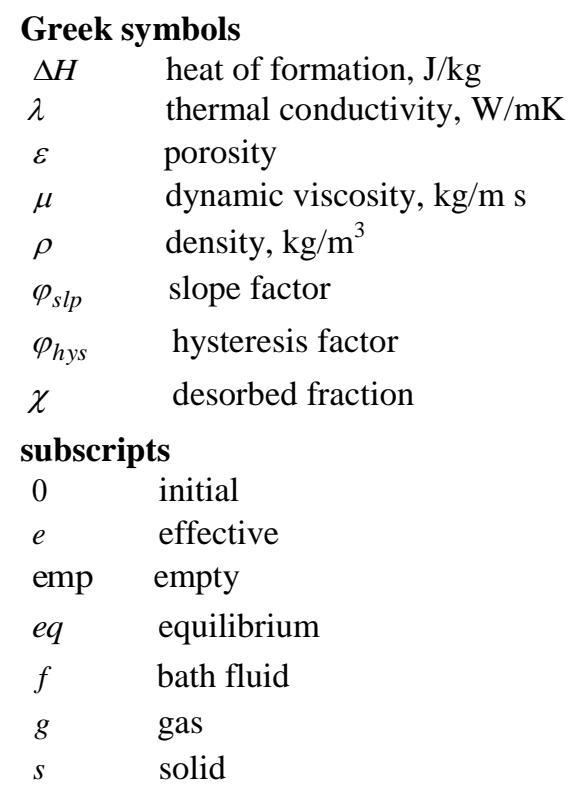




\section{INTRODUCTION}

Besides the fact that hydrogen is been sought as a clean alternative energy source, naturally abundant and having various production sustainable sources, hydrogen energy exploit encounter many industrial obstacles that are mainly categorized under transportation and/or storage related to the automotive industry. Among the techniques of storing hydrogen gas is to absorb it chemically by some simple or intermetallic compounds to form a metal hydride, this technique is qualified as solid state hydrogen storage. Therefore, many research studies have been carried out to improve this storage technique, and to enhance its reliability and safety in different applications.

The technology of solid state hydrogen storage offer high volumetric density compared against many other techniques, but unfortunately has a low hydrogen absorption capacity [1]. Nevertheless, in addition to their disadvantages of weight and cost, metal hydrides have another disadvantage which is their limited kinetics because they release/absorb huge amounts of heat whereas hydriding/dehydriding process, the latter is mostly restricted by the hydride's thermal properties and related cooling or heating techniques respectively. In fact, his causes serious challenges to the thermal management of the hydriding/dehydriding process [2]. Hence, the aforesaid thermal management of metal hydride reactors encounters the complex phenomenon of coupled heat and mass transfer in porous media where all the three modes of heat transfer take place. Thus in order to describe this phenomenon in packed beds, a variety of mathematical models have been reported on the heat and mass transfer in literature through multiple investigations[3-8], clarifying at which the amount of heat is removed or furnished to the metal hydride managed system.

These research investigations argued the obvious dependency of the hydrogen absorption and desorption rates on heat and mass transfer and on the reaction kinetics of the packed hydride beds [3-5]. Starting from one-dimensional models and focusing on heat conduction [6], afterward considering both convection and conduction [7-9], followed by coupling the conduction and convection with thermal radiation effects [10]. Effects of porous bed reactors geometries also have been studied notoriously; the two-dimensional models [8-9, 11-12] and the well-known three-dimensional study of Aldas et al. [3]. It was concluded from several works that radiative effects on the sorption process are negligible in the case of the $\mathrm{LaNi}_{5^{-}}$ based systems (low-temperature hydrides), and are important for the $\mathrm{Mg}$-based systems (high-temperature hydrides) [11], whereas the effect of convection is important and significantly influences the temperature repartition, however the overall hydride formation is found to be not affected by fluid flow [3]. Various hydride packed bed studies proved that twodimensional models are plenty adequate to represent heat and mass transfer in cylindrical shaped reactor beds (named metalhydride tanks), and can be employed in an enhanced bed-reactors design to reduce absorption and desorption times.

By numerically studying the solid state hydrogen storage in packed beds, Dogan et al. [13] found that the temperature increases adversely affect the absorption rate. Therefore the system must be efficiently cooled for a rapid charge. Furthermore, they investigated the effect of two charging methods on filling time, and found that hydrogen gas must be supplied radially for a fast filling operation. In their work, MacDonald and Rowe [14] examined, by mean of the resistive analysis calculations, the impacts of external convection resistance on thermodynamic behavior inside the MH tank. They used a one-dimensional resistive analysis and a two-dimensional transient model to determine the impact of external fins addition on the ability of a metal hydride tank to deliver hydrogen at a specified flow rate. They found that external fins addition have a large impact on the hydrogen gas pressure within the tank when a periodic hydrogen demand is imposed for the particular metal hydride alloy $\left(\mathrm{LaNi}_{5}\right)$ employed and the tank geometry studied. According to model results, they suggested that the metal hydride alloy at the tank core region can be removed to reduce weight and cost, without detrimental effects on the system performance.

Some widely-studied hydrogen storage alloy (like intermetallic compound) showed evidently that their hydrogen storage capacity $(w t . \%)$ is substantially lower than the current US Department of Energy (DOE) target for on-board hydrogen storage applications $[8,15]$. While, the introduction of the Computational Fluid Dynamics (CFD) techniques in the researches field of thermal engineering, have developed the design of heat exchange devices, particularly when investigating heat and mass transfer in porous media e.g. in packed bed reactors used for energy storage [10] or fuel cell applications modeling [16]. In their work dated in 2006, Marty et al. [4] aimed to numerically predict the coupled heat and mass transfer characteristics of an industrial cylindrical tank during hydrogen storage, using the commercial CFD code FLUENT. They emphasized the importance of achieving a three-dimensional modeling for the design of an industrial tank; their results were obtained on a magnesium hydride laboratory tank. Also, Phate et al. [5] deemed to explain hydride reactor coupled heat and mass transfer during absorption of hydrogen in cylindrical coordinates using the aforementioned CFD tool, and considering the effect of bulk diffusion to accurately model the mass transfer in solid phase. Both latter work models ignored the effects of plateau slope and the pressure-concentration isotherms (PCT) hysteresis related to the hydriding alloy used.

The simulation results of Mohan et al. [17] showed that the bed thickness is the major controlling parameter of the hydrogen absorption rate at all locations within the bed, although the heat exchanger (cooling tubes) dimensions are observed to have marginal influence on hydriding time of the $\mathrm{LaNi}_{5}$-based hydrogen storage device. Afterward, Krokos et al. [18] presented a systematically novel approach aiming to the design optimization of a multi-tubular metal hydride tank, containing up to nine tubular metal hydride reactors, and used in on-board hydrogen storage applications. They numerically investigated the arrangement of cooling tubes inside the metal hydride bed, founding that a uniform arrangement configuration of the embedded cooling tubes yielded to an optimal design parameter, consequently to their detailed three-dimensional mathematical model validated against that developed by Kikkinides et al. [12]. Thus by choosing a symmetric arrangement of the cooling tubes inside the reactor bed, Freni et al. [19] concluded that a shorter refueling time would be obtained at higher heat transfer rate. They have performed their numerical computations using the COMSOL Multiphysics simulation 
environment to demonstrate the fact that an increase in the hydride thermal conductivity can further improve dynamically the reactor performance, provided that the absorbent bed is sufficiently permeable to hydrogen.

Optimizing the performance and well designing the metal hydride $(\mathrm{MH})$ reactors is of primordial importance for the efficient operation of related systems in many industrial applications beside the hydrogen storage, particularly heat pumps and thermal compressors, rendering the configuration design an extremely imperative part in the MH reactors applications field.

Based on the aforesaid, we present this current work to emphasize the numerical study results of an Annulus-Disc Reactor (ADR) unit containing a packed hydrogen-saturated metal hydride alloy. In which a mass transfer takes place between the packed metal hydride and the hydrogen gas ceded to the inner tube of the disc unit. Concurrently, heat transfer occurs between the annulus-disc unit on one hand, and the heating fluid outside it and the tubes inside it, on the other hand. In $\mathrm{MH}$ applications, there are several types of reactors; the one studied in the present work enters under the shell and tube class type of heat exchangers studied by many researchers $[8,20]$. A practical configuration of an ADR would consist of many annular discs to store or provide hydrogen gas depending on whether hydrogen is being absorbed or desorbed. In this paper, we attempt to present a numerical study of such a device filled with the Mischmetal $\mathrm{MmNi}_{4.6} \mathrm{Fe}_{0.4}$ as the hydriding/dehydriding alloy.

Throughout the computations of this numerical investigation we have used OpenFOAM ${ }^{\circledR}$ [25], an open source finite volume code capable of solving industrial type problems, while adopting the two-dimensional mathematical model of Demircan et al. [11] to show and analyze the influence of the heat exchange device and the solid (MH) conductivity on dehydriding temperature fields of the $\mathrm{MH}$ bed reactor, and on the overall hydrogen gas desorption too.

This paper is organized as follows. Section 2 presents the mathematical model. Section 3 discusses the numerical method and techniques of resolution, which have been used to achieve computations. Section 4 presents the validation of the adopted model and numerical techniques, then the results discussion. Finally, a conclusion is given.

\section{MATHEMATical MODEL}

The mathematical model for the annulus-disc reactor (represented schematically in Figure 1) considered in the present work is similar to that of Demircan et al. [11], with $\mathrm{MmNi}_{4.6} \mathrm{Fe}_{0.4}$ as metal hydride alloy which is filled in the annular space between the inner central tube and the outer peripheral heating wall. The annulus disc is traversed by a number of active heating tubes in this case of the hydrogen desorption process. The thermo-physical properties of the alloy are as it is listed in Table 1. Because of angular symmetry we will restrict the computational domain to the sector area between two adjacent heating tubes. The computational domain accompanied with the mesh used in computations are shown in Figure 2.

Table 1. Thermo-physical properties of the metal hydride, hydrogen and other parameters used in the simulations [8, 14, 22 - 23].

\begin{tabular}{|l|l|}
\hline Parameters & Values \\
\hline Absorption rate constant $C_{d}$ & $475 \mathrm{~s}^{-1}$ \\
Specific heat of hydrogen gas $C_{p g}$ & $14.9 \mathrm{~kJ} / \mathrm{mol} \mathrm{K}$ \\
Specific heat of the solid $C_{p s}$ & $419 \mathrm{~J} / \mathrm{kg} \mathrm{K}$ \\
Activation energy for absorption $E_{d}$ & $25 \mathrm{~kJ} / \mathrm{mol}$ \\
Heat convection coefficient $h$ & $1652 \mathrm{~W} / \mathrm{m}^{2} \mathrm{~K}$ \\
Permeability of the metal $K$ & $10^{-8} \mathrm{~m}^{2}$ \\
Heat conductivity of the $\mathrm{H}_{2}$ gas $\lambda_{g}$ & $0.24 \mathrm{~W} / \mathrm{mK}$ \\
Heat conductivity in the metal $\lambda_{s}$ & $1.6 \mathrm{~W} / \mathrm{mK}$ \\
Molecular mass of hydrogen $M_{H_{2}}$ & $2.016 \mathrm{~kg} / \mathrm{kmol}$ \\
Porosity of the metal $\varepsilon$ & 0.5 \\
He-free metal hydride density $\rho_{e m p}$ & $8500 \mathrm{~kg} / \mathrm{m}^{3}$ \\
Reaction heat of formation $\Delta H$ & $27.5 \mathrm{~kJ} / \mathrm{kg}$ \\
Plateau slope $\varphi_{s l p}$ & 0.35 \\
Outlet pressure of hydrogen $P_{0}$ & $8 \mathrm{bars}$ \\
Outlet temperature of hydrogen $T_{0}$ & $298 \mathrm{~K}$ \\
HTF and tubes temperature $T_{f}$ & $298 \mathrm{~K}$ \\
Annular disc unit radius $R$ & $0.2 \mathrm{~m}$ \\
\hline
\end{tabular}




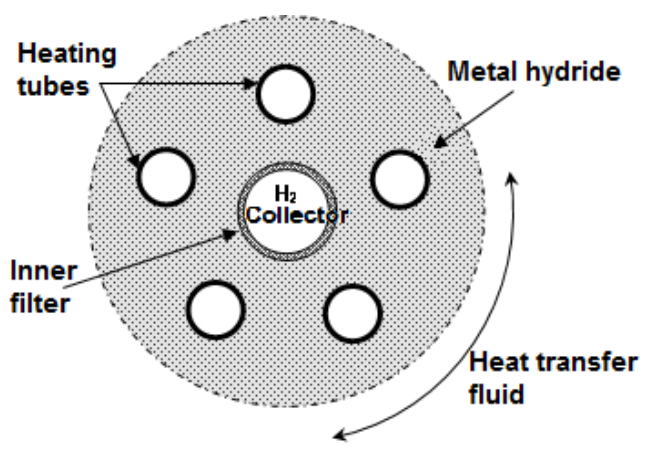

Fig. 1. Schematic section view of the studied ADR unit disc equipped with 5 heating tubes

The unit disc reactor considered is composed of a solid phase (metal-powder) and a gaseous phase (hydrogen), hence forming a porous medium. In order to establish the governing equations of the coupled heat and mass transfer with chemical reaction within the metal hydride reactors, we consider to up-scale these latter equations from pore level to macro level.

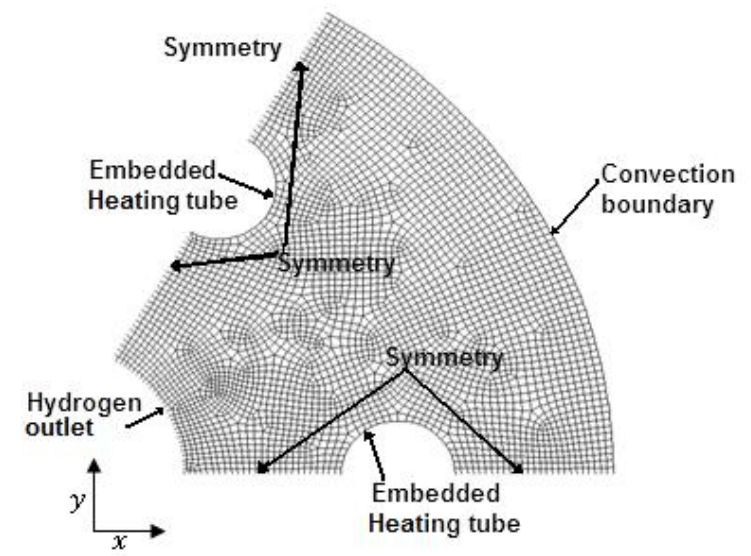

Fig. 2. One of the computational domains considered, meshed with the prescribed boundary conditions.

The main simplifying assumptions considered in applying the mathematical model are the following:

1. The media are in local thermal equilibrium between gas and solid

2. Radiative heat transfer is negligible.

3. The gas phase is ideal from the thermodynamic viewpoint.

4. Mass transfer takes place through the porous filter $\left(\mathrm{H}_{2}\right.$ collector $)$, while the hydrogen outlet temperature and pressure are maintained constants.

5. Porosity is uniform and its variation with desorption is negligible.

6. Equilibrium gas pressure in the bed obeys van't Hoff law relations.

7. Thermo-physical properties of both gas and solid phases are constant.

8. The radial temperature variation of the heating tubes is negligible; hence they are considered isothermal tubes.

\subsection{Volume-averaged mass balance equations}

\section{- For the hydrogen gas}

For describing the hydrogen flow and the desorption in a metal hydrogen reactor, unsteady flow is assumed. And the local hydrogen gas density $\rho_{g}$ and the local metal hydride density $\rho_{s}$ change as the metal hydride cede its hydrogen gas progressively; therefore an additional source term is included in the right-hand side of the continuity equation to denote the appearing amount of hydrogen in the bed with time

$$
\varepsilon \frac{\partial \rho_{g}}{\partial t}+\frac{\partial}{\partial x}\left(\rho_{g} u\right)+\frac{\partial}{\partial y}\left(\rho_{g} v\right)=\dot{m}
$$

\section{- For the metal-hydride alloy}

To model the density of metal powder changes due to the desorption of hydrogen, a sink term is added to the right-hand side of the governing equation, to denote the amount of hydrogen that is out-flowing from the solid with time. Hence,

$$
(1-\varepsilon) \frac{\partial \rho_{s}}{\partial t}=-\dot{m}
$$




\subsection{Hydrogen gas density}

The gas density $\rho_{g}$ is deduced from the perfect gas law

$$
\rho_{g}=\frac{P M_{H_{2}}}{\mathfrak{R} T}
$$

where $M_{H_{2}}$ and $\mathfrak{R}$ denote the molecular mass of hydrogen and the universal gas constant respectively.

\subsection{Volume-averaged momentum equations for the gas}

Due to the presence of the solid matrix, a pressure drop will occur in flows through porous media (metal hydride powder bed in this case). Accordingly, to reveal this effect a supplementary sink term should be added to the momentum equations in $x$ and $y$ directions. The above mentioned pressure drop depends on the properties of the porous medium and is described by including the Forchheimer modification term in $x$ and $y$ momentum equations which reduces to Darcy's law if the gas velocity and viscous boundary effect are low. Thus,

- In the $x$-direction,

$$
\rho_{g} \frac{\partial u}{\partial t}=-\frac{\partial P}{\partial x}+\mu\left(\frac{\partial^{2} u}{\partial x^{2}}+\frac{\partial^{2} u}{\partial y^{2}}\right)-\rho_{g}\left(u \frac{\partial u}{\partial x}+v \frac{\partial u}{\partial y}\right)+S_{D x}
$$

- In the $y$-direction,

$$
\rho_{g} \frac{\partial v}{\partial t}=-\frac{\partial P}{\partial y}+\mu\left(\frac{\partial^{2} v}{\partial x^{2}}+\frac{\partial^{2} v}{\partial y^{2}}\right)-\rho_{g}\left(u \frac{\partial v}{\partial x}+v \frac{\partial v}{\partial y}\right)+S_{D y}
$$

where $S_{D x}=-\left(\frac{\mu}{K} u+\rho_{g} b|\vec{V}| u\right)$ and $S_{D y}=-\left(\frac{\mu}{K} v+\rho_{g} b|\vec{V}| v\right)$ are the source terms instead of the additional pressure drop in the bed due to viscous effects and the increasingly important role played by fluid inertia. In these equations $|\vec{V}|$ is the magnitude of the hydrogen flow velocity. The following correlation for $b$ have been yielded from experimental measures implying the drainage of the gas through the columns of packed spheres, some sand and some pulverized coal (Bejan and Kraus [21]):

$$
b=\frac{1.75}{\sqrt{150 K \varepsilon^{3}}}
$$

where $K$ and $\varepsilon$ are the permeability and the porosity of the packed bed.

\subsection{Volume-averaged energy equation}

The energy equation in the metal bed can be expressed in terms of a single temperature variation, in this way we can conclude that the gas and solid phases quickly reach thermal equilibrium for the Mischmetal alloy used in the reactor [22]. Since dehydriding process inside the bed is an endothermic reaction, an amount of heat is consumed; subsequently an additional source term is added in the energy equation. Hence,

$$
\left(\rho C_{p}\right)_{e} \frac{\partial T}{\partial t}=\frac{\partial}{\partial x}\left(\lambda_{e} \frac{\partial T}{\partial x}\right)+\frac{\partial}{\partial y}\left(\lambda_{e} \frac{\partial T}{\partial y}\right)-\left(\rho_{g} C_{p g} u\right) \frac{\partial T}{\partial x}-\left(\rho_{g} C_{p g} v\right) \frac{\partial T}{\partial y}-\dot{m}\left(\Delta H-T\left(C_{p g}-C_{p s}\right)\right)
$$

with the effective volumetric heat capacity:

$$
\left(\rho C_{p}\right)_{e}=\varepsilon \rho_{g} C_{p g}+(1-\varepsilon) \rho_{s} C_{p s}
$$

and the effective thermal conductivity is taken as :

$$
\lambda_{e}=\varepsilon \lambda_{g}+(1-\varepsilon) \lambda_{s}
$$

\subsection{Reaction kinetics}

The amount of hydrogen that is desorbed from the hydrogen-saturated solid with time is directly related to the reaction rate of the dehydriding process of the metal, the latter is expressed as [11]:

$$
\dot{m}=C_{d} \exp \left(-\frac{E_{d}}{\Re T}\right)\left(\frac{P-P_{e q}}{P_{e q}}\right)\left(\rho_{s}-\rho_{e m p}\right)
$$

where $C_{d}$ is a material-dependent desorption rate constant, $E_{d}$ is the activation energy, $\rho_{\text {emp }}$ is the density of the empty metal hydride and $P_{e q}$ is the equilibrium pressure calculated using the van't Hoff relationship; 


$$
\ln \left(\frac{P_{e q}}{P_{0}}\right)=A-\frac{B}{T}+\varphi_{s l p}\left(\chi-\chi_{0}\right)+\varphi_{\text {hys }}
$$

where $\varphi_{s l p}$ is a factor which takes into account the slope of the plateau pressure in the PCT of hydriding/dehydriding metal alloy, and $\varphi_{\text {hys }}$ accounts for hysteresis between the absorption and desorption processes, but since the present work focus only on desorption process we will not take the hysteresis parameter into consideration. $P_{0}$ is the reference pressure (taken 1 atmosphere in all cases), $A$ and $B$ are van't Hoff constants having the values of 12.95 and 3731.42, respectively [23].

\subsection{Initial and boundary conditions}

Initially, the pressure, temperature and hydride density are assumed to be constant in the bed. In mathematical parlance, these conditions are written as:

At $t=0 ; \quad P(x, y, 0)=P_{0}, \quad T(x, y, 0)=T_{0}, \rho_{s}(x, y, 0)=\rho_{s 0}$.

For $t>0$;

- At the hydrogen outlet (the inner central tube) :

$$
P(x, y, t)=P_{0}, \quad T(x, y, t)=T_{0},
$$

- The lateral heating wall (peripheral wall) (Fig. 2) :

$$
-\lambda_{e} \frac{\partial T}{\partial \vec{n}}(x, y, t)=h\left(T_{f}-T\right)
$$

where $h$ is the heat transfer conductance between the metal hydride bed and the heating fluid having the temperature $T_{f}$ inside the peripheral jacket, taken from $[8-9,11]$, and $\vec{n}$ is the outward normal unit vector of the considered boundary.

- The embedded heating tubes, which are supposed to be isothermal (Fig. 2):

$$
T(x, y, t)=T_{f},
$$

- The other boundary conditions are assumed to have the symmetry condition type.

$$
\frac{\partial T}{\partial \vec{n}}(x, y, t)=0
$$

\section{NUMERICAL METHOD OF SOLUTION}

The governing equations are solved with a fully implicit finite volume numerical method implemented in the CFD OpenFOAM ${ }^{\circledR}[25]$. The solution procedure by means of the aforesaid method consists of transforming the governing differential equations into a general algebraic form, after integrating those numerically over a finite volume-meshed computational domain (Versteeg and Malalasekera [24]). A transport equation in the general form may be written as

$$
\frac{\partial(\rho \Phi)}{\partial t}+\vec{\nabla} \cdot(\rho \vec{V} \Phi)=\vec{\nabla} \cdot\left(\Gamma_{d} \vec{\nabla} \Phi\right)+S_{c}
$$

where $\Phi$ denotes a generic transported variable quantity, $\Gamma_{d}$ denotes the diffusion coefficient and $S_{c}$ the source term. This common form equation is solved using the pressure-velocity coupling algorithm named SIMPLEC [24] which give to our CFD calculations more rapidity, stability and consistency, knowing that the Second Order Upwind scheme is used in the purpose of interpolating momentum convective fluxes [24-25]. Calculations were carried out on a Core ${ }^{\mathrm{TM}}$ i5 PC, leading to take about 8 hours per a typical case, while using highly optimized compilation for the binaries.

Statistically, the volume-weighted average of the problem physical quantities defined by the following relation:

$$
\bar{\Phi}=\frac{\sum_{i} \sum_{j} \Phi_{i, j} V_{i, j}}{\sum_{i} \sum_{j} V_{i, j}}
$$

provides a reliable measure of the $\mathrm{MH}$ reactor performance, where $\Phi_{i, j}$ is the generic variable quantity, corresponding to the control volume $V_{i, j}$, in the sense of finite volume method [8].

\section{RESULTS AND DISCUSSION}

\subsection{Grid independency tests}

After adopting the mathematical model, and establishing the relevant solution procedure, a mesh independency tests were performed to examine the effect of the computational mesh on the dynamical behavior of the average reacted fraction. 
Various quadrilateral mesh grids for the considered domain geometry were exploited in the independency test calculations, consisting in structured and non-structured types. It was observed a close accord between the several tested computational meshes, which concede to the present simulations a clear independency from the computational meshes.

\subsection{Validation of numerical results}

First of all, before showing and discussing the obtained results, we have carried out some simulations for the validation of the considered model in comparison with some experimental data present in the literature, mainly those from the work of Jemni et al. [22], in which a cylindrical shaped $\mathrm{LaNi}_{5}-\mathrm{H}_{2}$ reactor was investigated. Also we have assumed axial symmetry to apply the adopted two-dimensional approach to deal with the three-dimensional experimental reality. Figure 3 shows the temperature time-evolution inside the metal bed in three different points within the reactor. The temperature of the heating fluid and the outlet pressure are $T_{f}=20^{\circ} \mathrm{C}$ and $P=8 \mathrm{bars}$ for the absorption case, respectively. At the beginning of the desorption process, the temperatures show a rushed decrease in the metal bed, because the hydrogen-metal reaction is endothermic then increase gradually with the reaction kinetics exponential growth. The simulation results were in excellent agreement with the experiments, conceding that the adopted model can be used for further investigations of the transient thermal-fluid behavior in $\mathrm{MH}$ reactors.

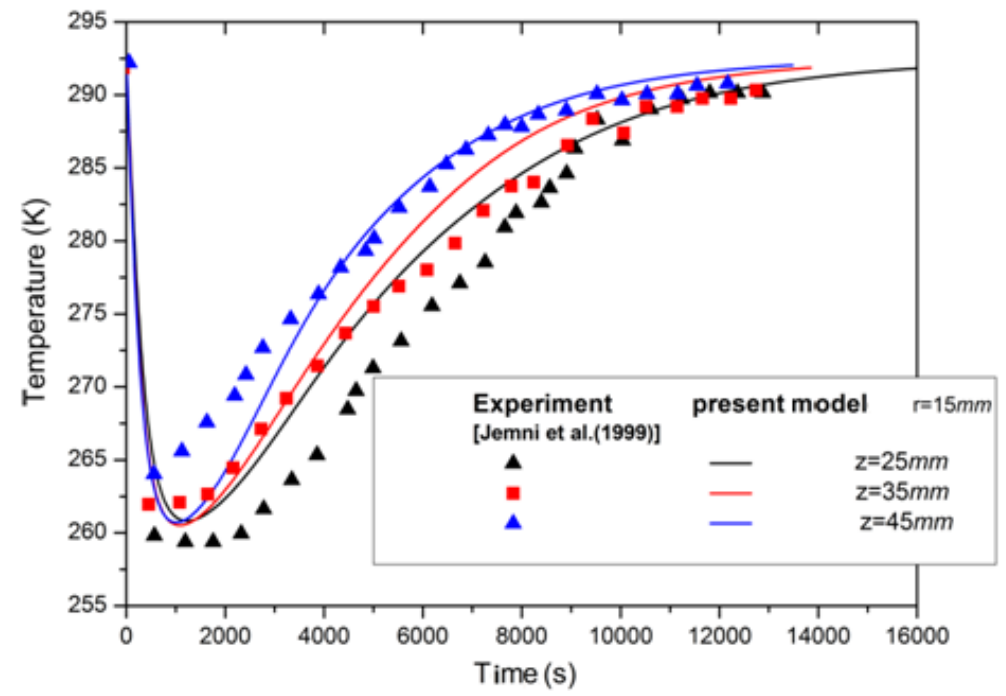

Fig. 3. Temperature evolution at three points within the cylindrical MH tank experimented by Jemni et al. [22] in the hydriding case with the heating fluid temperature at $20^{\circ} \mathrm{C}$

\subsection{Hydrogen desorption from the $\mathrm{MmNi}_{4.6} \mathrm{Fe}_{0.4}$ alloy}

We focus our attention in this section impacts of heat transfer fluid temperature, and metal alloy thermal conductivity on hydrogen gas desorption inside the ADR-type metal-hydride reactor. Thus, the heat and mass transfer dynamical behavior inside the annulus-disc reactor is examinated by means of several numerical simulations of the packed bed containing the Mischmetal $\mathrm{MmNi}_{4.6} \mathrm{Fe}_{0.4}$, considered as hydrogen-saturated $\left(\mathrm{MmNi}_{4.6} \mathrm{Fe}_{0.4} \mathrm{H}_{6}\right)$. This $\mathrm{AB}_{5}$-type alloy, which present equilibrium pressures ranging from 10 to 20 bars at the ambient temperature, and can stock up to 1.6 wt\% reversibly.

Desorption numerical simulations presented in this paper were carried at a constant discharging pressure of 35 bars, and at temperature of $25^{\circ} \mathrm{C}$. Simulations are realized for the heating temperature range ; $T_{f}=20^{\circ}, 25^{\circ}, 30^{\circ}$ and $35^{\circ} \mathrm{C}$, in the aim to inspect the heating temperature influence on the hydrogen desorption time.

Storage metal thermal conductivity effects on the ADR reactor performance in discharging time terms, and related to the $\mathrm{H}_{2}$ reacted fraction, are examined by carrying a set of numerical simulations ranging in the interval; $\lambda_{s}=2.4,3,5 \mathrm{et} 8 \mathrm{~W} / \mathrm{mK}$.

\subsubsection{HTF temperature Effects}

Initially, the system is discharged at an outlet pressure of 35 bars and outlet temperature of $25^{\circ} \mathrm{C}$. The corresponding hydrogen concentration is $1.57 \mathrm{wt} \%$.

Figure 4 gathers the time evolutions of the hydrogen desorption capacity (wt\%), calculated inside the MH reactor at the monitoring point $\mathrm{S}_{3}(0.092,0.094)$. We can observe from these time history curves that hydrogen desorption capacity is always an increasing function monotonically, until reaching the limit capacity. Hence, the HTF temperature augmentation reduces visibly the discharging time of the $\mathrm{H}_{2}$ gas. Consequently, the heating temperatures affect positively the examined $\mathrm{MH}$ reactor performance enhancements in desorption. 


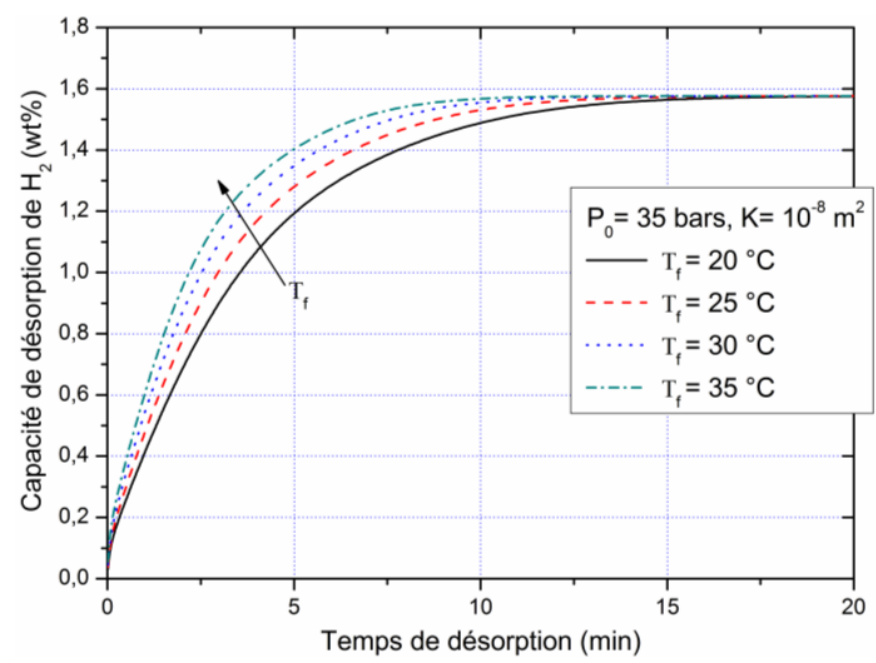

Fig. 4 Temporal evolution of the storage capacity of the packed bed of $\mathrm{MmNi}_{4.6} \mathrm{Fe}_{0.4}$ at point $\mathrm{S}_{3}(0.092,0.094)$.

From the Figure 4, we observe also that the required time to attain the limit value of desorption capacity is considerably short ( $<15$ minutes). This can be explained by the fact that the monitoring point $\left(\mathrm{S}_{3}\right)$ position is located near the heated walls.

Figure 4 showing the temporal evolution curves of the average desorbed fraction, affirms the conclusion that the heating temperature augmentation is a favorable parameter in the $\mathrm{MH}$ reactor desorption performance.

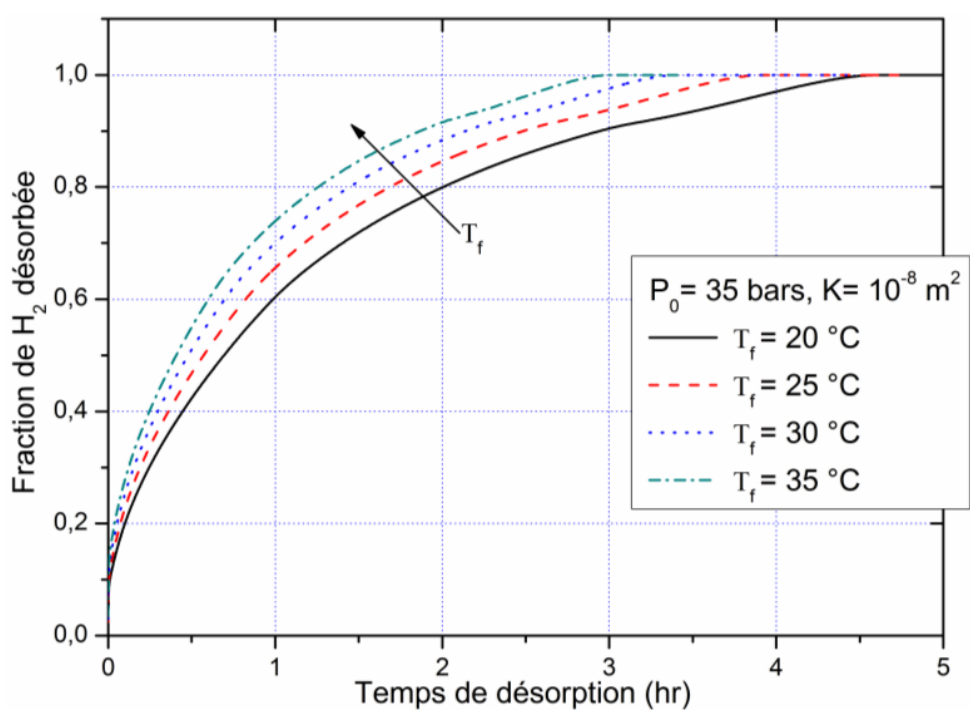

Fig. 5 HTF temperature effect on $\mathrm{H}_{2}$ desorption at constant discharging pressure.

The average desorbed fraction plots show that a complete desorption takes a delay of 4.5 hours in the case of the $20{ }^{\circ} \mathrm{C}$ heating temperature-reactor, nevertheless the same average fraction requires only about 3 hours to be desorbed completely, which represents $33 \%$ of the reaction time reduction.

Obviously, from the profile plots of hydrogen desorption capacity along the midline, at instants indicated on Figure 6 , we can mention the conclusion that a wave-like (in motion) behavior of the desorbed fraction take place within the MH reactor. Figure 6 confirms again this conclusion, and announces that a complete dehydridation (desorption) is achieved after 3 hours for the case of heating temperature of $308 \mathrm{~K}$. 


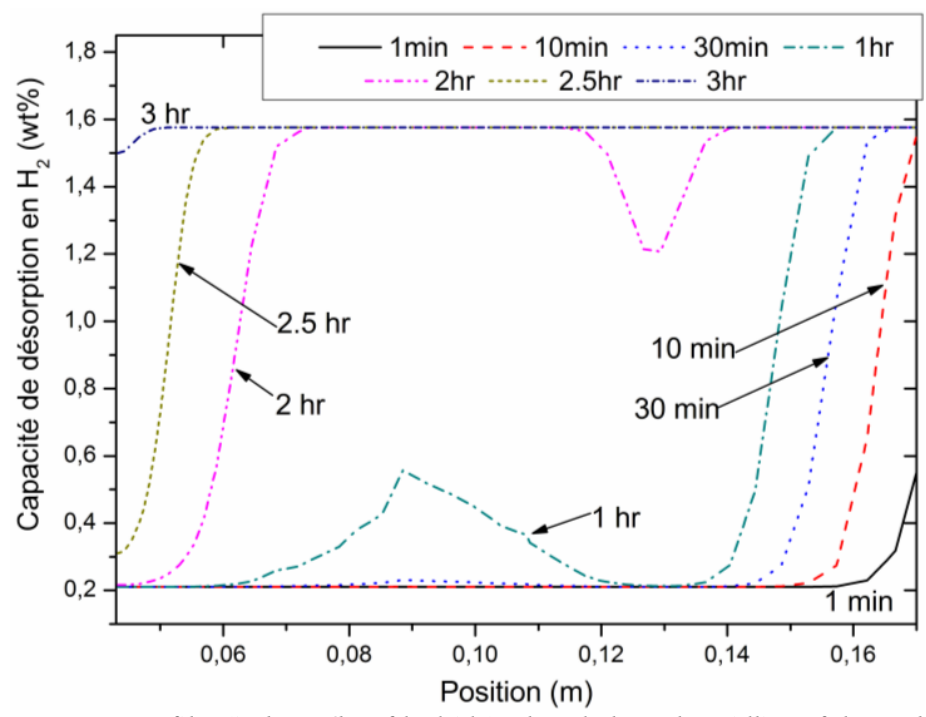

Fig. 6 Desorbed hydrogen amount profiles (in $\mathrm{kg} \mathrm{H}_{2} / \mathrm{kg}$ of hydride), plotted along the midline of the packed bed, at selected times.

\subsubsection{Metal-hydride thermal conductivity effect}

The $\mathrm{MH}$ bed thermal conductivity is a key-parameter in enhancing reactor performance. Figure 7 shows the temporal evolutions of the hydride bed average temperature obtained by numerical simulations for different thermal conductivities. As expected, a thermal conductivity increase could be directly interpreted in a discharging (desorption) time decrease within the $\mathrm{MH}$ reactor. Practically, the results obtained for this packed bed reactor type incites us to investigate the MH reactors design containing highly conductive porous matrices, or to incorporate several heat exchangers devices inside the metal-hydride bed [16]. Moreover, the average density of the metal hydride is affected by its thermal conductivity, as we can observe on Figure 8 showing thermal conductivity effect on solid (metal-hydride) average density, where thermal conductivity increase involves a substantial $\mathrm{H}_{2}$ discharging time reduction, knowing that for the metal thermal conductivity of $8 \mathrm{~W} / \mathrm{mK}$, the solid cede the entirety hydrogen absorbed mass in a time period of 1.5 hour. Reacted fraction values $\chi$ (desorbed amount) close to unity indicate high desorption rate, which conduct to conclude that hydrogen desorption process take place essentially in hot regions inside the reactor, or near walls undergoing thermal exchange (heating). The core region of the $\mathrm{MH}$ reactor having the weakest reaction rate, which yet has drawn to the suggestions to take-off this region without harmful effect on reactor performance [14].

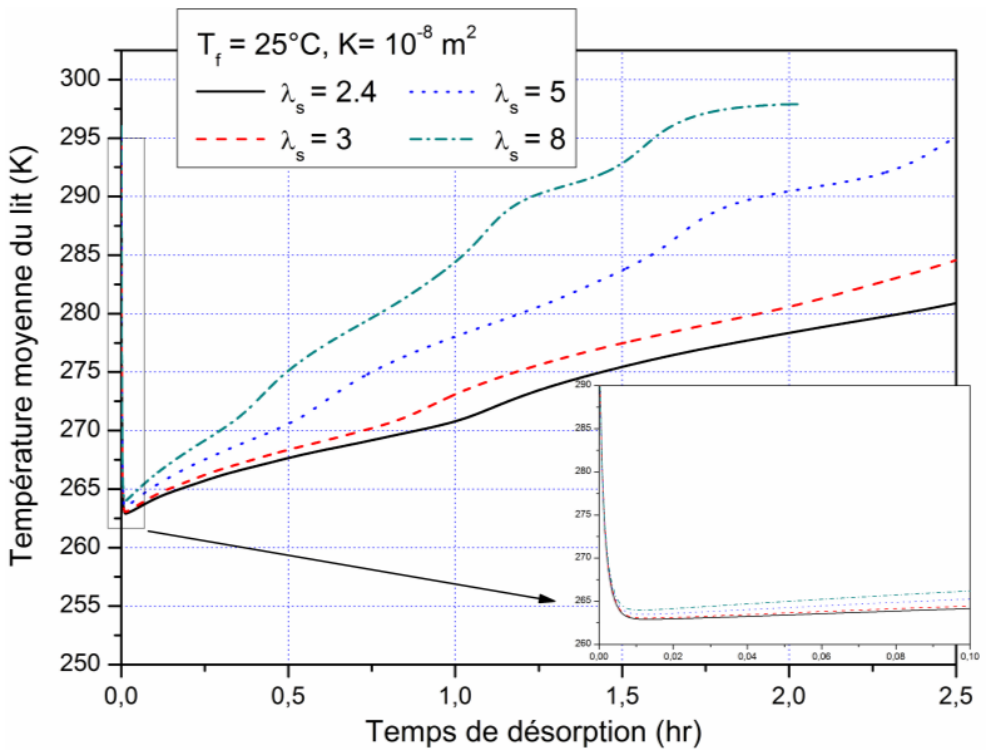

Fig. 7 Temporal evolutions of desorption temperatures for different $\mathrm{MH}$ thermal conductivities. 


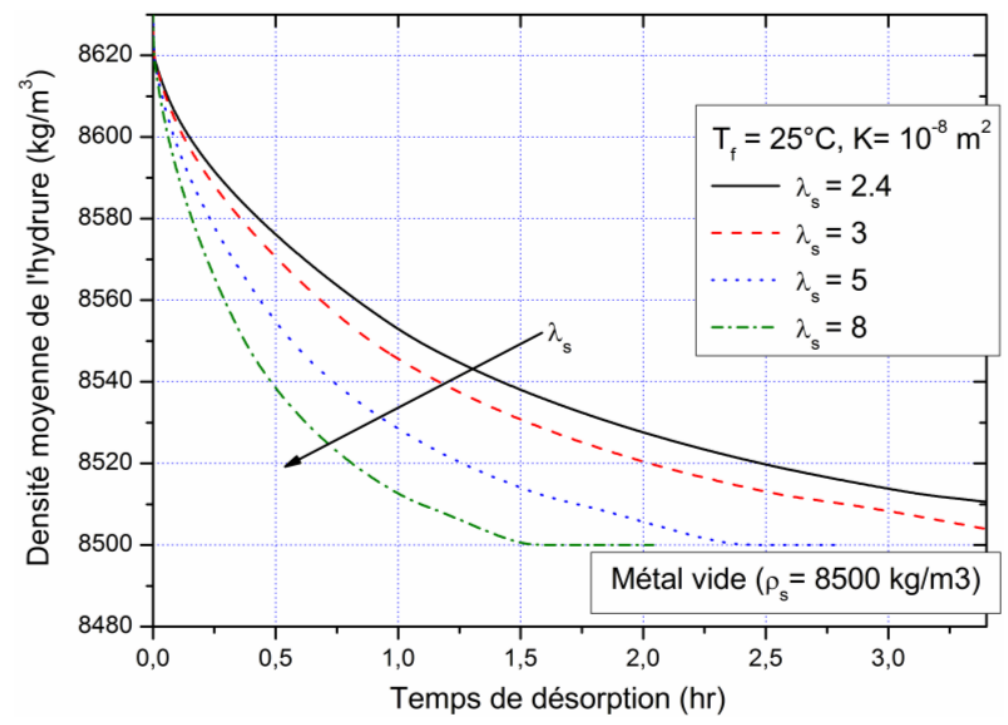

Fig. 8 Thermal conductivity effect on $\mathrm{MH}$ density during $\mathrm{H}_{2}$ desorption.

\section{CONCLUSION}

In this work, the hydrogen gas desorption in metal hydride bed is numerically investigated in an annulus-disc reactor unit. A two-dimensional mathematical model to describe the transient coupled heat and mass transfer in the hydride bed during the desorption process of hydrogen has been adopted and employed. The calculations were performed using the finite volume method bundled in the open CFD software called OpenFOAM ${ }^{\circledR}$, whilst taking into account the PCT's plateau slope factor of the dehydriding alloy (its kinetics), and the additional pressure drop in the bed caused by fluid inertia (momentum), besides considering the Forchheimer modification source terms in the momentum equations of the present mathematical model. The numerical results showed good accordance with the experimental data reported in the literature.

\section{REFERENCES}

[01] L. K. Heung, "Using metal hydride to store hydrogen,” DOE report, WSRC-MS-2003-00172, 2003.

[02] A. Isselhorst, "Heat and mass transfer in coupled hydride reaction beds," J. Alloys Compounds, vol. 231, pp. 871-79, 1995.

[03] K. Aldas, M. D. Mat and Y. Kaplan, "A three-dimensional mathematical model for hydrogen absorption in a metal hydride bed,” Int. J. Hydrogen Energy, vol. 27, pp. 1049-56, 2002.

[04] P. Marty, J. F. Fourmigue, P. De Rango, D. Fruchart and J. Charbonnier, "Numerical simulation of heat and mass transfer during the absorption of hydrogen in a magnesium hydride," Energy Conversion and Management, vol. 47, pp. 3632-43, 2006.

[05] A. K. Phate, P. Maiya and S. Murthy, "Simulation of transient heat and mass transfer during hydrogen sorption in cylindrical metal hydride beds," Int. J. Hydrogen Energy, vol. 32, pp. 1969-81, 2007.

[06] R. Gopal and S. Murthy, "Prediction of heat and mass transfer in annular cylindrical metal hydride beds," Int. J. Hydrogen Energy, vol. 17, pp. 795-805, 1992.

[07] H. Choi and A. F. Mills, "Heat and mass transfer in metal hydride beds for heat pump applications," Int. J. Heat and Mass Transfer, vol. 33, pp. 1281-89, 1990.

[08] A. Boukhari and R. Bessaïh, "Numerical heat and mass transfer investigation of hydrogen absorption in an annulusdisc reactor", Int. J. Hydrogen Energy, vol. 40(39), pp. 13708-17, 2015.

[09] D. Chabane, F. Harel, A. Djerdir, M. Ibrahim, D. Candusso, O. Elkedim and N. Fenineche, "Influence of the key parameters on the dynamic behavior of the hydrogen absorption by LaNi,", Int. J. Hydrogen Energy, (Article in press), 2016.

[010] I. A. El-Osery, M. A. El-Osairy, A. M. Metwally, M. M. Keshk and M. El-Gammal, "Dynamic simulation of the convective model for metal hydride hydrogen storage beds," Energy Sources, vol. 15, pp. 523-30, 1993.

[011] A. Demircan, M. Demiralp, Y. Kaplan, M. D. Mat and T. N. Veziroglu, "Experimental and theoretical analysis of $\mathrm{H}_{2}$ absorption in $\mathrm{LaNi}_{5}-\mathrm{H}_{2}$ reactors," Int. J. Hydrogen Energy, vol. 30, pp. 1437-46, 2005.

[012] E. Kikkinides, M. Georiadis and A. Stubos, "On the optimization of hydrogen storage in metal hydride beds," Int. J. Hydrogen Energy, vol. 31, pp. 737-51, 2006.

[013] A. Dogan, Y. Kaplan and T. N. Veziroglu, "Numerical investigation of heat and mass transfer in a metal hydride bed," App. Math. and Computation, vol. 150, pp. 169-80, 2004. 
[014] B. MacDonald and A. Rowe, "Impacts of external heat transfer enhancements on metal hydride storage tanks," Int. J. Hydrogen Energy, vol. 31, pp. 1721-31, 2006.

[015] D. P. Broom, Hydrogen storage materials: The characterization of their storage properties, Springer-Verlag London, 2011.

[016] M. Visaria and I. Mudawar, "Experimental investigation and theoretical modeling of dehydriding process in highpressure metal hydride hydrogen storage systems," Int. J. Hydrogen Energy, vol. 37, pp. 5735-49, 2012.

[017] G. Mohan, P. Maiya and S. Murthy, "Performance simulation of metal hydride hydrogen storage device with embedded filters and heat exchanger tubes," Int. J. Hydrogen Energy, vol. 32, pp. 4978-87, 2007.

[018] C. A. Krokos, D. Nikolic, E. S. Kikkinides, M. C. Georgiadis and A. K. Stubos, "Modelling and optimization of multitubular metal hydride beds for efficient hydrogen storage," Int. J. Hydrogen Energy, vol. 34, pp. 9128-40, 2009.

[019] A. Freni, F. Cipiti and G. Cacciola, "Finite element-based simulation of a metal hydride-based hydrogen storage tank,” Int. J. Hydrogen Energy, vol. 34, pp. 8574-82, 2009.

[020] F. Yang, X. Meng, J. Deng, Y. Wang and Z. Zhang, "Identifying heat and mass transfer characteristics of metal hydride reactor during adsorption- parameter analysis and numerical study," Int. J. Hydrogen Energy, vol. 33, pp. 1014-22, 2008.

[021] A. Bejan and A. D. Kraus, Heat Transfer Handbook, John Wiley \& Sons, Inc, 2003.

[022] A. Jemni, S. Ben Nasrallah and J. Lamloumi, "Experimental and theoretical study of a metal-hydrogen reactor," Int. J. Hydrogen Energy, vol. 24, pp. 631-44, 1999.

[023] G. Sandrock and G. Thomas, IEA/DOE/SNL Hydride Databases, [Online available] http://hydpark.ca.sandia.gov.

[024] H. K. Versteeg and W. Malalasekera, An Introduction to Computational Fluid Dynamics: the finite volume method, $2^{\text {nd }}$ Edition, Pearson Education Ltd, 2007.

[025] OpenV FOAM, The Open Source CFD Toolbox User Guide, Version v1606+, OpenCFD Ltd, 2016. 